\title{
Aqueous and Methanol Extracts of Paullinia pinnata (Sapindaceae) Improve Monosodium Urate-Induced Gouty Arthritis in Rat: Analgesic, Anti-Inflammatory, and Antioxidant Effects
}

\author{
Pius Pum Tseuguem, Télesphore Benoît Nguelefack (D), Basile Ngnanmegne Piégang, \\ and Sorelle Mbankou Ngassam \\ Research Unit of Neuro-Inflammatory and Cardiovascular Pharmacology, \\ Laboratory of Animal Physiology and Phytopharmacology, University of Dschang, P.O. Box 67, Dschang, Cameroon \\ Correspondence should be addressed to Télesphore Benoît Nguelefack; nguelefack@yahoo.fr
}

Received 28 August 2019; Revised 10 October 2019; Accepted 22 October 2019; Published 5 December 2019

Guest Editor: Samuel Martins Silvestre

Copyright (c) 2019 Pius Pum Tseuguem et al. This is an open access article distributed under the Creative Commons Attribution License, which permits unrestricted use, distribution, and reproduction in any medium, provided the original work is properly cited.

\begin{abstract}
The profound modification of lifestyle and food habits has led to an important increase in the prevalence of gout. Unfortunately, there are current unmet needs for the treatment of this disease, prompting the search for new alternatives. Paullinia pinnata is a plant used to treat various diseases including arthritis. The present work aimed to investigate the antigouty activities of the aqueous (AEPP) and methanolic (MEPP) extracts of $P$. pinnata as well as their in vivo antioxidant properties. The gouty arthritis was induced by injecting $50 \mu \mathrm{l}$ of monosodium urate (MSU, $100 \mathrm{mg} / \mathrm{ml})$ in the left hind ankle of rats. $P$. pinnata extracts were administered orally at the doses of 100 and $200 \mathrm{mg} / \mathrm{kg} /$ day for 6 days, starting $24 \mathrm{~h}$ after MSU injection. Allopurinol $5 \mathrm{mg} / \mathrm{kg} / \mathrm{day}$ was used as reference drug. Inflammation and hyperalgesia were daily monitored from 24 hours after treatment initiation and for the 6 consecutive days. Myeloperoxidase (MPO) quantification was done in collected synovial fluid. Nitrite oxide (NO), malondialdehyde (MDA), and superoxide dismutase (SOD) were evaluated in the spinal cord and the brain. The serum content of SOD was additionally quantified. AEPP and MEPP significantly $(p<0.001)$ reduce MSU-induced inflammation $(22.41 \%$ to 93.65\%) and hyperalgesia (33.33\% to 64.44\%) in both ankle and paw. AEPP and MEPP significantly $(p<0.001)$ reduce synovial MPO production with the percentage ranging from $76.30 \%$ to $85.19 \%$. AEPP and MEPP significantly $(p<0.001)$ reduce serum, spinal, left and right hemispheres NO, and MDA and increase the SOD activity $(p<0.001)$. $P$. pinnata leaf extracts possess potent curative effects against MSU-induced gouty arthritis that combines analgesic, anti-inflammatory, and antioxidant activities. These findings support the use of $P$. pinnata leaves extracts in the treatment of gouty arthritis and further present the plant as a potent source of efficient antigouty medicine.
\end{abstract}

\section{Introduction}

Gouty arthritis is a painful inflammatory disease, characterized by a swollen and painful joint which usually extends to the surrounding tissue. Gouty arthritis usually occurs following hyperuricemia, identified as the major risk factor $[1,2]$. This joint disorder is characterized by the precipitation, deposition, and crystallization of monosodium urate in the joints, leading to intense inflammation and painful process [3]. The gout prevalence ranges between
4 and $6.8 \%[4,5]$. Its incidence is constantly rising, and it is associated with many comorbidities. In the development of the pathology, the deleterious effects of urate are primarily due to its capacity to trigger the production of reactive oxygen species (ROS), thus promoting the oxidative status in gouty arthritis patients. Concordantly, oxidative stress status has been demonstrated in gouty arthritis [6].

The timeline treatment against gout focuses on the treatment of inflammation and the reduction of hyperuricemia. Many agents, including allopurinol, febuxostat, 
colchicine, nonsteroidal anti-inflammatory drugs, corticosteroids, and more recently interleukin (IL)-1 inhibitors, have been developed and have demonstrated efficiency in gout treatment. Nevertheless, they have some limitations among which are little effect on acute gout attacks, contraindications with the comorbidities, and drugs' side effects, which include fever, skin rashes, allergic reactions, hepatitis, nephropathy, and gastrointestinal toxicity/bleeding [7-9]. There is, therefore, an important need for more effective new therapeutics with low side effects.

One of the most reliable sources of new compounds is made of medicinal plants. They have gained more interest these last decades and are now intensively used by industries for the production of phytopharmaceuticals and nutraceuticals.

The search for new natural drugs with antigouty effects, combining anti-inflammatory, analgesic, and antioxidant properties, is the focus of this manuscript. Paullinia pinnata Linn is a creeper plant (Sapindaceae) widely distributed in the west and the center regions of Cameroon. It is empirically used for the treatment of infection and painful and inflammatory diseases. The leaves decoction is used in the East Africa region, for the treatment of snake bites [10]. Phytochemical studies of this plant extract revealed the presence of phenols [11], flavonoids, triterpene, saponins and tannins [12], steroids, steroidal glycosides, and cerebroside and ceramide [13]. Compounds such as 2 -O-methyl-L-chiro-inositol, $\beta$-sitosterol, and friedelin have been isolated from P. pinnata [14]. Previous pharmacological studies demonstrated the antimicrobial activities and protective effects against CCl4-induced hepatic damage and oxidative stress $[14,15]$. Our recent studies showed that the aqueous and methanol extracts from the leaves of $P$. pinnata possess potent preventive effects on rheumatoid monoarthritis [16]. But whether these plant extracts can cure gouty arthritis is unknown. The present study was undertaken to evaluate the curative effect of the aqueous and methanol extracts of $P$. pinnata on the MSU-induced gouty arthritis in Wistar rats.

\section{Materials and Methods}

2.1. Plant Material and Preparation of Extracts. The fresh leaves of $P$. pinnata were harvested at Poumougne in the Koung-khi Division (West Region, Cameroon) in March 2017. The identification of plant specimens was done at the Cameroon National Herbarium in Yaoundé by Mr. Tadjouteu Fulbert in comparison with a voucher specimen under the reference number 10701/SRF. Cam.

The leaves were shade dried and ground into a fine powder. The aqueous extract was prepared by decoction. The powder $(500 \mathrm{~g})$ was boiled in $3.5 \mathrm{~L}$ of distilled water for 15 minutes. The mixture was filtrated on Whatman paper No. 1 . The residue was re-extracted as previously described in $2 \mathrm{~L}$ of distilled water for 15 minutes. The two filtrates obtained were pooled together and evaporated in a ventilated steam room for 72 hours at $40^{\circ} \mathrm{C}$ to yield $89.54 \mathrm{~g}(17.91 \%)$.

The methanol extract was prepared as maceration. The powder $(500 \mathrm{~g})$ was soaked in $3.5 \mathrm{~L}$ of methanol for 72 hours and filtrated using Whatman paper No. 1. The plant material residue was re-extracted in $2 \mathrm{~L}$ of methanol for 24 hours. The two filtrates obtained were mixed and concentrated in a rotary evaporator under low pressure at $40^{\circ} \mathrm{C}$ and yielded $111.04 \mathrm{~g}(22.21 \%)$ of methanol extract.

Both aqueous and methanol extracts were prepared in $4 \%$ DMSO for the experiment prior to administration to animals.

2.2. Chemicals and Drugs. Uric acid, hexadecyltrimethylammonium bromide, epinephrine, O-dianisidin, and sodium azide were purchased from Sigma-Aldrich Chemical Co. (Taufkirchen, Germany). Sodium hydroxide, hydrochloric acid, bovine serum albumin, sodium chloride, and Dimethylsufoxide (DMSO) were purchased from CarlRoth (Kalshur, Germany). Nitric acid was purchased from Qualikems. Allopurinol was obtained from Aspen-Pharma (Ireland). Ether was purchased from a local pharmacy (Dschang, Cameroon). Griess reagent, Biuret reagent, and carbonate buffer were prepared in the laboratory with ingredients from Sigma-Aldrich Chemical Co. (Taufkirchen, Germany). Thiobarbituric acid was purchased from Shanghai Zhanyun Chemical Co. Ltd (China). Trichloroacetic acid was purchased from Guangdon Guanghua SciTech Co. Ltd (China).

\subsection{Monosodium Urate (MSU) Crystal Synthesis. MSU} crystals were synthesized according to a modified protocol of Tank et al. [17]. Two-hundred milliliters of an aqueous solution of $\mathrm{NaOH}(1 \mathrm{M})$ was stirred well and heated up to $70^{\circ} \mathrm{C}$. After obtaining the required temperature, $1 \mathrm{~g}$ of uric acid was added into the $\mathrm{NaOH}$ solution and $\mathrm{HCl}(1 \mathrm{~N})$ was then used to maintain the mixture at $\mathrm{pH}$ 7.2. The solution was stirred at a constant temperature $\left(70^{\circ} \mathrm{C}\right)$ for 4 hours and then stirred slowly at room temperature and stored at $4^{\circ} \mathrm{C}$ for $24 \mathrm{~h}$. Fine particles of MSU crystals were found at the bottom of the glass. MSU particles were recovered by filtration, washed with distilled water; air-dried, and suspended in sterile saline $(100 \mathrm{mg} / \mathrm{ml})$.

2.4. Animals. Albino Wistar rats $(150-200 \mathrm{~g})$ were bred onsite in the Laboratory of Physiology and Phytopharmacology of the University of Dschang, Cameroon. Forty-two animals (10 to 12 weeks old) of either sex were divided into 7 experimental groups of 6 animals each (3 males and 3 females). Rats were habituated to pain threshold recording and paw volume measurement before the experiment. Rats with baseline pain threshold below or equal to $7.5 \mathrm{~g}$ were discarded.

2.5. MSU-Induced Gouty Arthritis. The experiment was set following the method of Zhang et al. [18], with some modifications, and performed as it is described below. Briefly, rats were anesthetized with ether, the left hind leg skin was sterilized with $75 \%$ ethanol, and the lateral malleolus located by palpation. Then, an insulin syringe needle was inserted vertically to penetrate the skin and turned distally for insertion into the articular cavity at the gap 
between the tibiofibular and tarsal bone until a distinct loss of resistance was felt. Fifty microliters of MSU was injected into the articulation to induce gouty arthritis (day 0 ). The same procedure was followed for all the groups except for the sham group in which only sterile saline was injected in the articulation. One day after MSU injection, the second baselines of inflammation and pain threshold were taken. Then, the $6 \mathrm{MSU}$-injected groups were treated as follows: negative control treated orally with DMSO (4\% in distilled water), positive control treated with Allopurinol $(5 \mathrm{mg} / \mathrm{kg} /$ day; dissolved in distilled water), and the 4 remaining groups treated orally with AEPP or MEPP at the doses of 100 and $200 \mathrm{mg} / \mathrm{kg} /$ day. The MSU-injected rats were treated daily for 6 consecutive days. The pain threshold at the ankle and paw was recorded using an analgesimeter (Ugo Basile, type 37215) on days $1,2,3,4,5$, and 6 after the initiation of treatments. Briefly, awake rats were scruffed and placed in the analgesimeter. For each measuring time point, two trials were completed on the ipsilateral ankle and the values averaged. The same measurement procedure was conducted on the ipsilateral paw.

Immediately after pain threshold measurements, the paw and the ankle volumes were recorded using an electronic caliper (Fine Science, Heidelberg, Germany). Rats were slightly restrained, and the diameter of the paw was measured at the space between all footpads from the back (dorsal surface) to the front (plantar surface) of the paw and even the ankle.

On day 7 after MSU injection, animals were anesthetized by intraperitoneal injection of $15 \%$ ethyl carbamate solution $(1.5 \mathrm{~g} / \mathrm{kg})$. Blood, spinal cord, and the left and right hemispheres were collected for antioxidant assays. The synovial liquid of the injected ankle was collected by washing the joint with $100 \mu \mathrm{l}$ of $\mathrm{NaCl} 9 \%$ for the evaluation of myeloperoxidase level.

Blood collected in nonheparinized tubes was centrifuged at $3000 \mathrm{rpm}$, and the serum was kept at $-20^{\circ} \mathrm{C}$ until use. The entire spinal cord and the left and right hemisphere were homogenized in $10 \%$ and $15 \%$ solution of ice-cold $0.1 \mathrm{M}$ Tris buffer $(\mathrm{pH} 7.4)$ at $4^{\circ} \mathrm{C}$, respectively, and centrifuged at $10,000 \mathrm{rpm}$ at $4^{\circ} \mathrm{C}$ for $15 \mathrm{~min}$. The supernatant was collected and keep at $-20^{\circ} \mathrm{C}$. Serum, spinal cord, left and right hemisphere supernatants were used for superoxide dismutase (SOD), nitrite oxide (NO), and malondialdehyde (MDA) estimation.

2.6. Nitric Oxide, Superoxide Dismutase, and Malondialdehyde Assays. The Griess reagent was used to estimate the nitric oxide content. To $250 \mu \mathrm{l}$ of the tissue sample was added $250 \mu \mathrm{l}$ of $1 \%$ sulfaniladide prepared in $5 \%$ orthophosphoric acid. The mixture was incubated for 5 minutes in the dark, then $250 \mu \mathrm{l}$ of $0.1 \%$ naphthyl ethylenediamine was added, and then all incubated in the dark for additional 5 minutes. The optical density was read at $530 \mathrm{~nm}$. The quantity of nitric oxide was calculated from the sodium nitrite's standard curve.

The SOD activity assessment was conducted according to the protocol of Wandji et al. [19]. To $70 \mu \mathrm{l}$ of the sample was added $830 \mu \mathrm{l}$ of carbonate buffer $(\mathrm{pH}=10.2)$ and $100 \mu \mathrm{l}$ of epinephrine $(0.3 \mathrm{mM})$. The mixture was homogenized, and the absorbance read 60 and 120 seconds after epinephrine was introduced, at $480 \mathrm{~nm}$ using a spectrophotometer (Helios Epsilon). The inhibitory percentage of oxidation was calculated according to the following formula:

$$
I \%=100-\left[\frac{\text { Ab sample }}{\text { Ab blank }}\right] \times 100,
$$

where $I \%=$ inhibitory percentage and $\mathrm{Ab}=$ absorbance.

The SOD activity was calculated as:

$$
A=\frac{I \%}{50 \times \text { protein quantity }}, \text { with AA }=\text { the SOD activity, }
$$

$50=$ one unit.

For the MDA assay, $500 \mu \mathrm{l}$ of orthophosphoric acid (1\%) and $500 \mu \mathrm{l}$ of thiobarbituric acid (1\%) were added to $100 \mu \mathrm{l}$ of serum/supernatant. The mixture was homogenized and placed in boiling water bath for 15 minutes. Thereafter, the tubes were cooled on ice bath and centrifuged at $3000 \mathrm{rpm}$ for 10 minutes. The supernatant was collected, and the absorbance was read at $532 \mathrm{~nm}$ using a spectrophotometer (Helios Epsilon). The concentration of MDA was calculated based on the absorbance coefficient according to the following formula: D. O. $=\varepsilon$. C. L (where D. O.=optical density, $\varepsilon=$ molar extinction coefficient of the MDA $\left(1.56 \times 10^{5} \mathrm{M}^{-1} . \mathrm{Cm}^{-1}\right), \quad C=$ concentration in MDA, and $L=$ length of the optic journey $(1 \mathrm{~cm}))$.

2.7. Myeloperoxidase Assay. This assay was conducted as previously described by Garça et al. [20], with some modifications. In each well of a 96-well plate, $25 \mu$ l of the synovial liquid, $25 \mu \mathrm{l}$ of potassium phosphate buffer, and $25 \mu \mathrm{l}$ of $0.5 \%$ hexadecyltrimethylammonium bromide buffer were introduced. The mixture was incubated for 30 minutes at room temperature. Then, $10 \mu \mathrm{l}$ of sodium azide $(0.1 \mathrm{mM})$ and $150 \mu \mathrm{l}$ of potassium phosphate buffer (50 mM; pH 5.5) containing $0.026 \%$ ortho-dianisidine dihydrochloride and $0.018 \%$ hydrogen peroxide were added to the reaction medium. The entire plate was once again incubated at room temperature for 30 minutes. Finally, the optical density of each well of the 96-well plate was read using an ELIZA microplate reader at $450 \mathrm{~nm}$. MPO level was expressed using an arbitrary unit.

2.8. Statistical Analysis. Results are expressed as mean \pm SEM. Time-dependent hyperalgesia and inflammation were analyzed using two-way ANOVA followed by Bonferroni's posttest while time point data were analyzed with one-way ANOVA followed by Tukey's posttest. All the analyses were performed with GraphPad Prism 5.01 software package. A $p$ value of less than 0.05 was considered as statistically significant. 


\section{Results}

3.1. Anti-Inflammatory Effects of P. pinnata Extracts on MSUInduced Gouty. Intra-ankle injection of MSU resulted in a significant inflammation that was maintained for at least 6 days. Daily oral administration of $P$. pinnata extracts significantly reduced the inflammation induced by MSU both at the level of the ankle and the paw. Two-way ANOVA (main effects time, treatment, and interaction) demonstrated significant effects at all the doses used $(p<0.05 ; p<0.01$; $p<0.001$ ) (Figures 1(a)-1(d)). Roughly, both AEPP and MEPP showed dose-dependent effects. At the dose of $200 \mathrm{mg} / \mathrm{kg}$, AEPP and MEPP exhibited maximal inhibitory percentages of 99.23 and $98.40 \%$, respectively. The inhibitory percentage induced by allopurinol $(5 \mathrm{mg} / \mathrm{kg} /$ day $)$ ranged from 26.76 to $90.81 \%$ and was, in general, less efficient than $P$. pinnata extracts (Figure 1).

3.2. Antihyperalgesic Effects of P. pinnata Extracts on MSUInduced Gouty. The intra-articular administration of MSU resulted in hyperalgesia both at the level of the ankle and the paw. The oral administration of $P$. pinnata extracts starting one day after MSU injection significantly reduced the mechanical hyperalgesia as measured with the Randall Sellito method (two-way ANOVA effects time, treatment, and interaction $p<0.01 ; p<0.001)$. As demonstrated by Bonferroni posttest, AEPP, and MEPP induced time- and dosedependent antihyperalgesic effects (Figures 2(a)-2(d)). MEPP at the dose of $200 \mathrm{mg} / \mathrm{kg}$ almost completely abolished the mechanical hyperalgesia, both at the level of the paw and the ankle (Figures 2(b) and 2(d)). The effect of allopurinol was equal to that of MEPP at the dose of $100 \mathrm{mg} / \mathrm{kg}$.

3.3. Inhibitory Effects of P. pinnata Extracts on the MPO Activity in Synovial Liquid in Gouty Rats. In the process of inflammation, neutrophils through their oxidative burst produce MPO. As shown in Figure 3, the intra-articular injection on MSU drastically increased $(p<0.001)$ the MPO activity in the synovial fluid as compared to the sham group. AEPP and MEPP significantly $(p<0.01 ; p<0.001)$ reduced the activity of MPO, with the highest effect observed with AEPP at the dose of $200 \mathrm{mg} / \mathrm{kg}$ (Figure 3(a)). The positive control allopurinol-treated group $(5 \mathrm{mg} / \mathrm{kg}$ ) (Figures 3(a) and $3(\mathrm{~b}))$ also significantly $(p<0.001)$ reduces the MPO level as compared to untreated MSU control and more efficiently than both AEPP and MEPP at both doses (100 and $200 \mathrm{mg} / \mathrm{kg})$.

3.4. P. pinnata Extracts Inhibit the NO Production in the Spinal Cord and the Right and Left Hemispheres of Gouty Rat. During the inflammatory process, immune cells play an essential role while defending the organism by producing nitrite oxide. For more insight on the anti-inflammatory activities of $P$. pinnata extracts, we evaluated the effect of AEPP and MEPP on the nitric oxide production in the spinal cord and the right and left hemispheres. The control group (MSU-DMSO) showed a marked increase $(p<0.001)$ in the
NO content in all the sample regions collected. This increase was significantly and completely reversed $(p<0.001)$ by $P$. pinnata extracts and allopurinol in all the collected organs and at all the doses used, as compared to the control group (Figures 4(a)-4(f)).

3.5. Effects of P. pinnata on the SOD Activity in the Serum, Spinal Cord, and Right and Left Hemispheres of Gouty Rat. The impact of $P$. pinnata extracts on oxidative stress was evaluated through the SOD activity measured in the serum, spinal cord, and right and left hemispheres. MSU ankle injection did not significantly affect the SOD activity in all the tissues used, as compared to the sham group. However, the daily administration of AEPP and MEPP induced a tissue-dependent increase in the SOD activity. In fact, $P$. pinnata extracts did not affect the evaluated parameter in the right hemisphere. Besides, MEPP showed no effect on the SOD activity in the left hemisphere (Figure 5(h)). Allopurinol was able to significantly increase the SOD activity only in the serum and the left hemisphere, while both extracts did so in the serum and the spinal cord. More, AEPP increases the parameter in the left hemisphere in a dose-dependent manner (Figure 5(g)).

3.6. P. pinnata Extracts Reduce the MDA Content in the Spinal Cord and Right and Left Hemispheres of Gouty Rat. MDA is the end-product of lipid peroxidation and can indicate the status of oxidative stress. To evaluate the protective effect of $P$. pinnata extracts against the oxidative damages in the pain modulating/integrative structures, we quantified the MDA content in the spinal cord and cerebral hemispheres. As expected, we found a significant $(p<0.001)$ increase of MDA content in MSU-injected rats as compared to the sham group (Figures 6(a)-6(f)). AEPP and MEPP significantly $(p<0.001)$ reduced the MDA content in the spinal cord and right and left hemispheres as compared to the MSU-control group. The positive control allopurinol also produces a significant $(p<0.001)$ effect, but this inhibitory activity was lower than that of both extracts independently of the tissue.

\section{Discussion}

The present study was undertaken to investigate the ability of the aqueous (AEPP) and methanol (MEPP) extracts of $P$. pinnata against pain, inflammation, and oxidative stress in gouty arthritis, a disease of the musculoskeletal system characterized by painful inflammatory joints and oxidatives. Gout is the result of the accumulation of monosodium urate (MSU) crystals in the joint. Thus, experimental MSU-induced gouty arthritis is a trustworthy model of gout that mimics the clinical human condition. In the present study, the intra-ankle injection of MSU resulted in inflammation and hyperalgesia in both the ipsilateral ankle and paw, accompanied by an increase in MPO activity in synovial fluid and oxidative stress in the spinal cord and the brain. These results largely corroborate the previous findings [21-23]. 


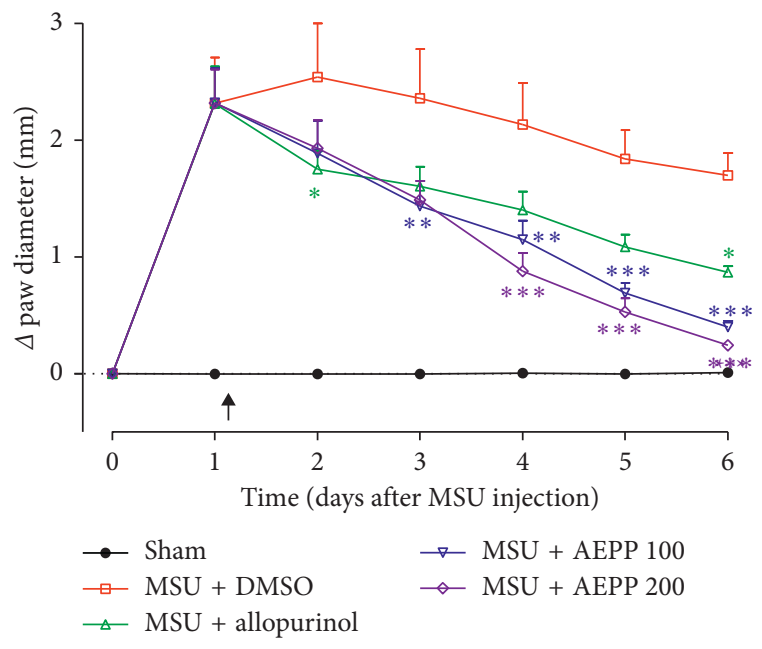

(a)

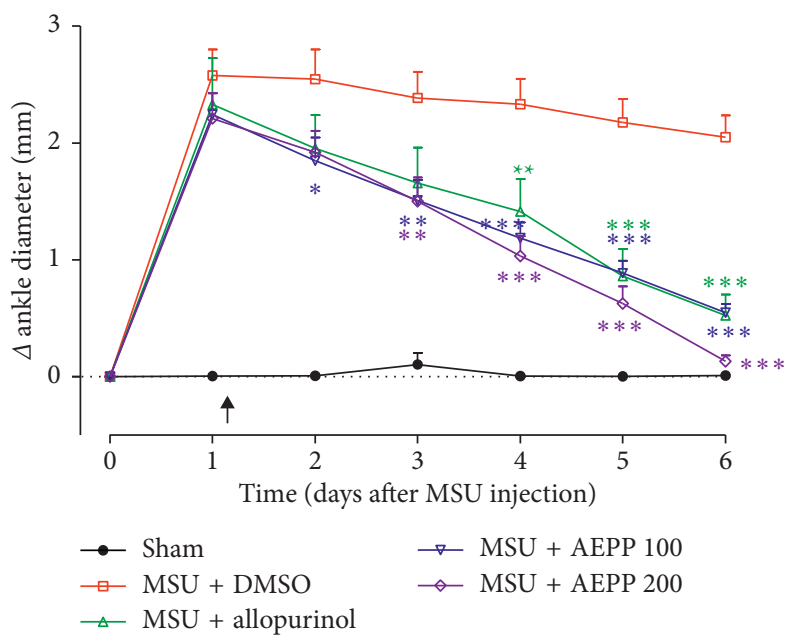

(c)

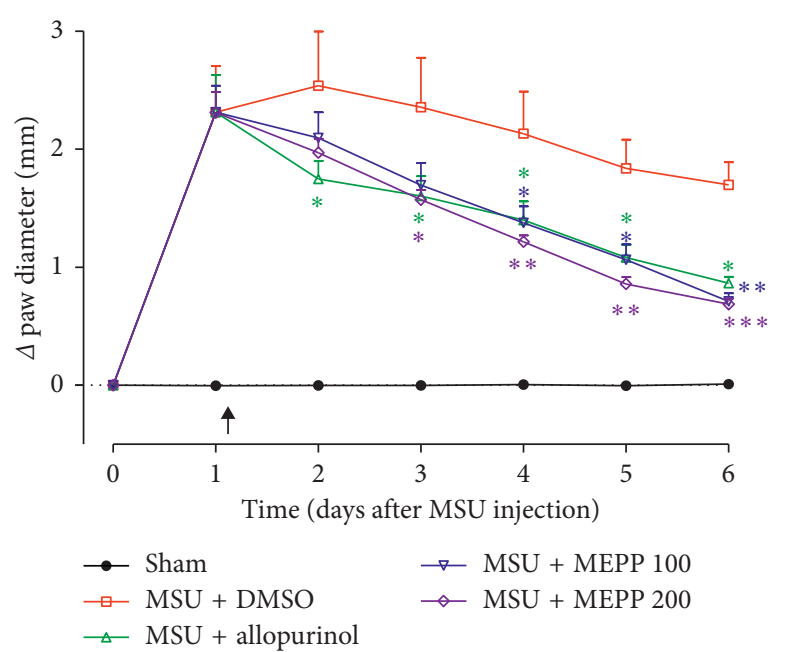

(b)

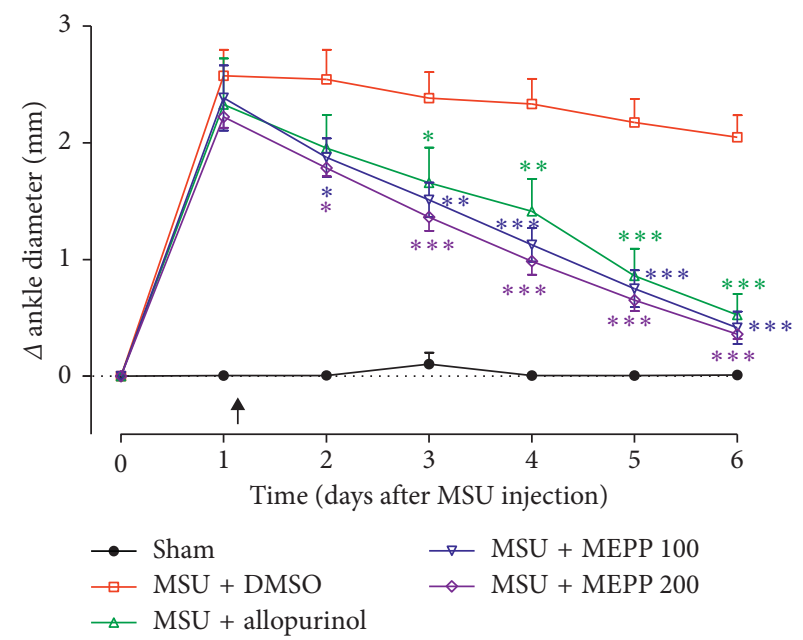

(d)

FIgUre 1: Effects of the (a, c) aqueous (AEPP 100, $200 \mathrm{mg} / \mathrm{kg} /$ day) and (b, d) methanolic (MEPP 100, 200 mg/kg/day) extracts of Paullinia pinnata on the primary (ankle $(a, b)$ ) and secondary (paw $(c, d)$ ) inflammatory gout induced by MSU ankle injection in rat. (a) AEPP induces significant decreases in ankle swelling compared to rats treated with MSU and vehicle (3\%DMSO). (b) MEPP induces significant decreases in ankle swelling compared to rats treated with MSU and vehicle. (c) AEPP induces significant decreases in paw swelling compared to rats treated with MSU and vehicle. (d) MEPP induces significant decreases in paw swelling compared to rats treated with MSU and vehicle. Allopurinol $(5 \mathrm{mg} / \mathrm{kg} /$ day $)$ is included as a positive control for anti-inflammatory effects. Each point represents the mean \pm SEM of 6 individual rats. ${ }^{* *} p<0.01,{ }^{* * *} p<0.001$; significant difference compared to the negative control group (MSU + DMSO) using two-way ANOVA with Bonferroni posttest. The black arrow indicates the beginning of treatments.

Oral administration of the AEPP and MEPP significantly reduced inflammation and hyperalgesia in a dose- and timedependent manner, reduced the myeloperoxidase activity in synovial fluid, and exhibited significant in vivo antioxidant effects on NO, MDA, and SOD.

Cumulative literature is concordant on the physiopathological process of MSU-induced gout. In fact, at the first step, phagocytes in the joint such as macrophages, monocytes, or neutrophils will activate upon detection of MSU crystals, to produce active IL- $1 \beta$ which will subsequently activate NF-KB to trigger the transcription of cytokines and chemokines as well as the release of inflammatory mediators. The released mediators will finally activate nociceptor endings, opening TRPV1 and TRPA1 channels either directly or through intracellular signaling cascades. Furthermore, the antidromic firing of articular nociceptors releases neuropeptides from peripheral nerve terminals, thereby potentiating the local inflammatory response known as neurogenic inflammation [24, 25]. As a consequence of these intricate pathways, there will be hypersensitization of nociceptors ending for hypernociception and allodynia, as well as inflammation in the joint and surrounding tissues. AEPP and MEPP showed time-dependent analgesic and anti-inflammatory effects, suggesting that these extracts possess bioactive molecules that may interfere with the different pathways described above. $P$. pinnata extracts may, therefore, act on phagocytes and directly on the nervous system. Previous studies with the same 


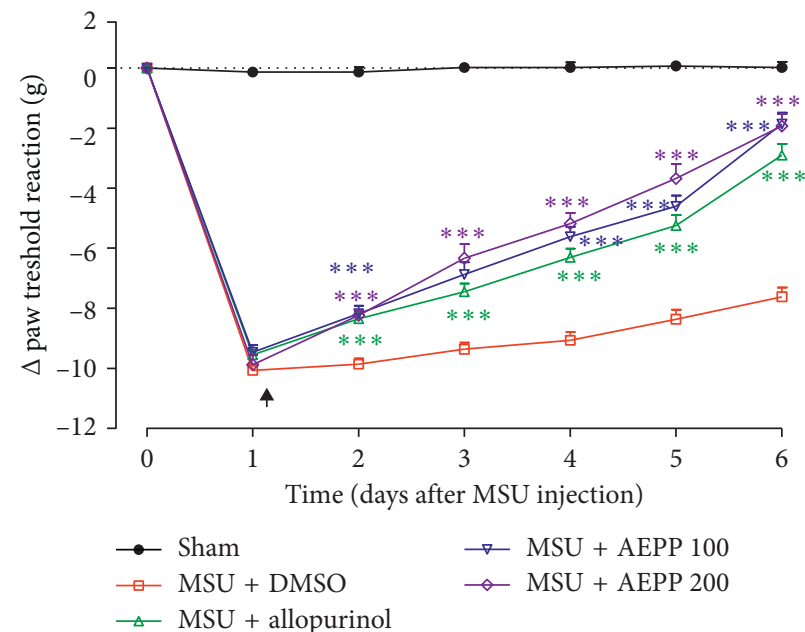

(a)

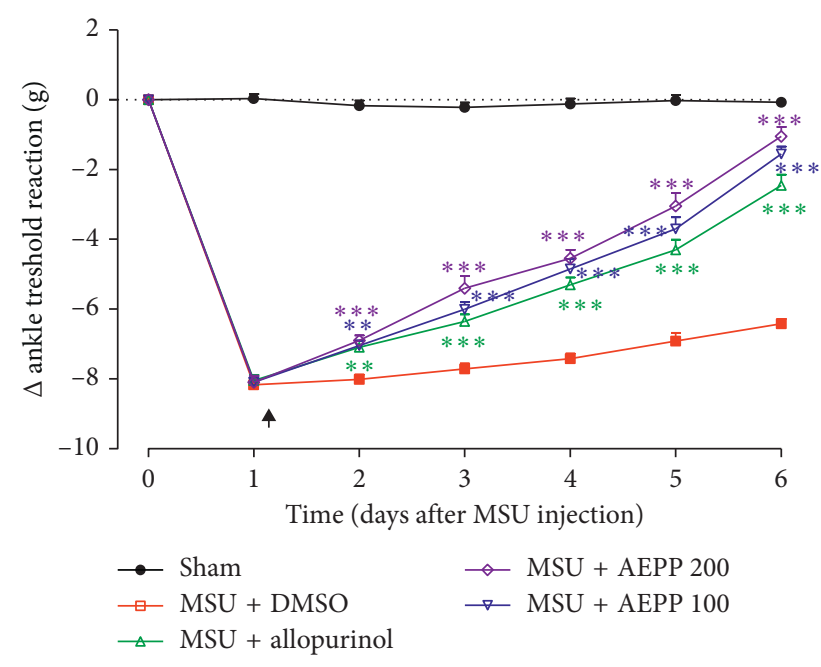

(c)

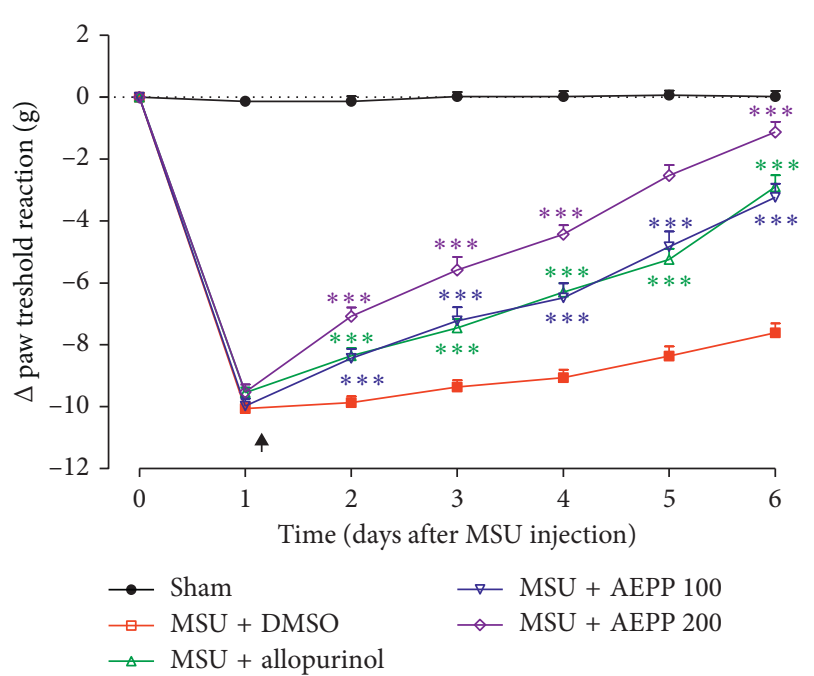

(b)

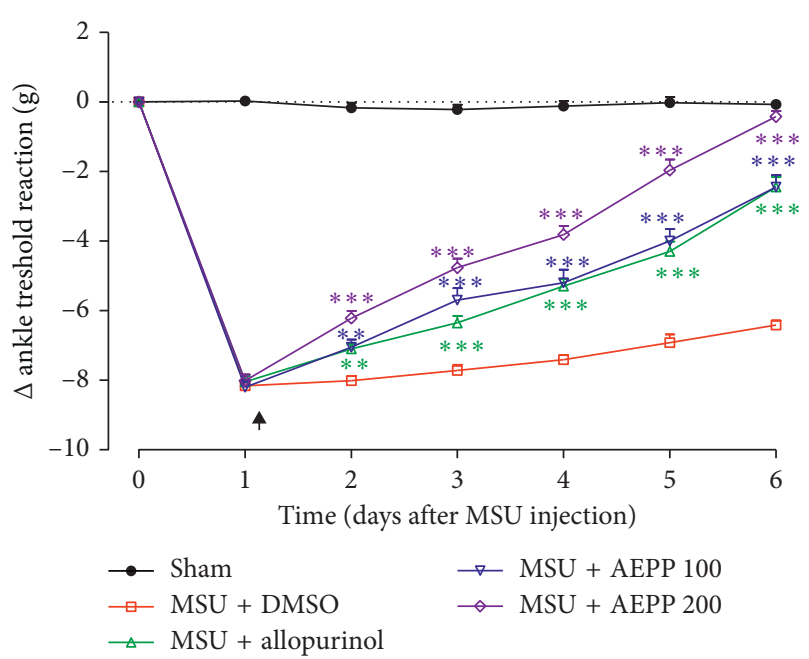

(d)

FIGURE 2: Effects of the aqueous (AEPP; 100, $200 \mathrm{mg} / \mathrm{kg} /$ day) and methanol (MEPP; 100, $200 \mathrm{mg} / \mathrm{kg} /$ day) extracts of Paullinia pinnata on the primary (ankle) and secondary (paw) mechanical hypersensitivity induced by ankle injection of MSU in rat. (a) AEPP induces significant decreases in mechanical hypersensitivity (i.e., decreased change in threshold from baseline) compared to rats treated with MSU and vehicle (3\%DMSO). (b) MEPP induces significant decreases in mechanical hypersensitivity compared to rats treated with MSU and vehicle. (c) AEPP induces significant decreases in mechanical hypersensitivity compared to rats treated with MSU and vehicle. (d) MEPP induces significant decreases in mechanical hypersensitivity compared to rats treated with MSU and vehicle. Allopurinol ( $5 \mathrm{mg} / \mathrm{kg} / \mathrm{day})$ is included as a positive control for the antihyperalgesic effect. Each point represents the mean \pm SEM of 6 individual rats. ${ }^{* *} p<0.01$, ${ }^{* * *} p<0.001$; significant difference compared to the negative control group (MSU + DMSO) using two-way ANOVA with Bonferroni posttest. The black arrow indicates the beginning of treatments.

extracts of $P$. pinnata demonstrated their analgesic and antiinflammatory effects in Complete Freund's Adjuvant- (CFA-) induced monoarthritis and suggested that the extracts may have effect both at the central and peripheral levels [16]. Besides, it was also demonstrated that these extracts inhibit the production of IL- $1 \beta$ and TNF- $\alpha$ by macrophages in inflammatory conditions. As IL- $1 \beta$ plays a pivotal role in the development of pain and inflammation in the context of hyperuricemia or MSU accumulation in the joint, it can be suggested that the analgesic and anti-inflammatory effects of $P$. pinnata extracts are related to their IL- $1 \beta$ inhibition capacity. As such, the analgesic effect of the plant extracts will be highly dependent on its anti-inflammatory properties.

Nevertheless, AEPP and MEPP were able to reduce the secondary analgesia at the level of the paw. This result suggests that $P$. pinnata extracts may act directly on the central nervous system, as secondary pain depends mostly on the sensitization of the central nervous system [26].

It has clearly been shown that neutrophil recruitment and activation are the cornerstones of the inflammatory response to monosodium urate (MSU) crystals [27]. Neutrophils infiltrated and accumulated in the synovial joint will undergo degranulation and release their secretion products 


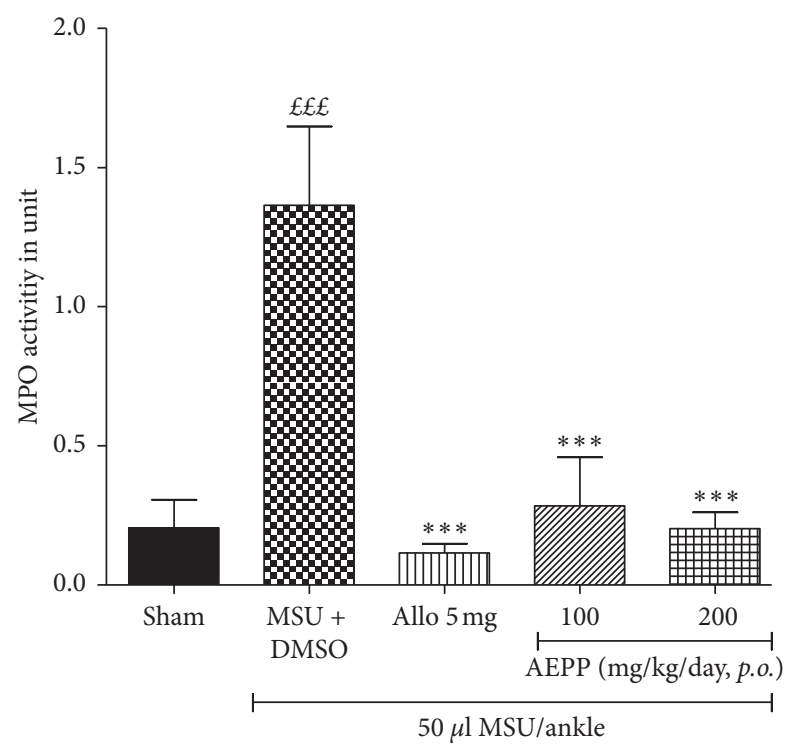

(a)

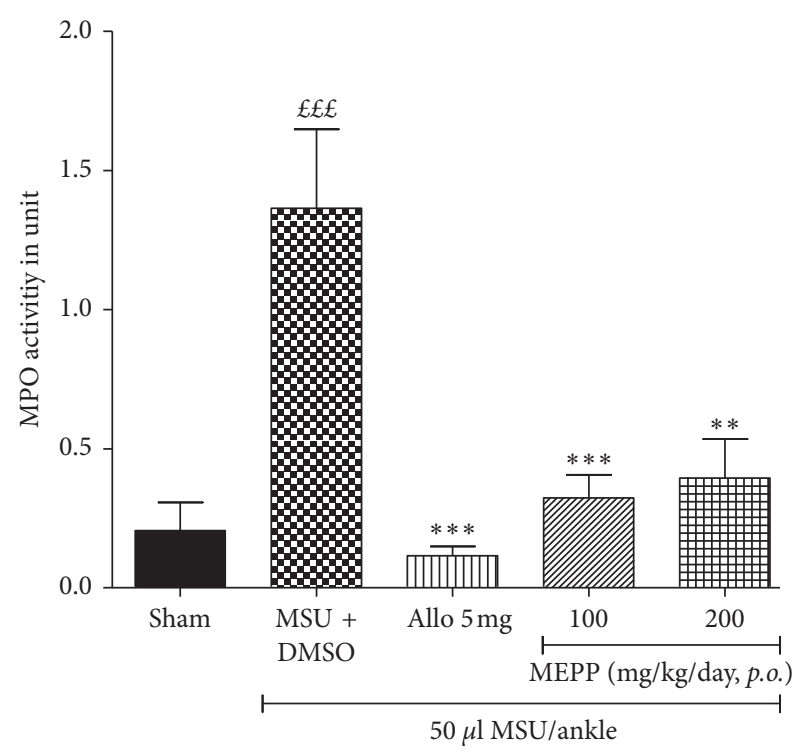

(b)

FIGURE 3: Effects of the aqueous (AEPP) and methanol (MEPP) extracts of Paullinia pinnata on myeloperoxidase (MPO) activity in the synovial liquid of MSU-injected animals. Allopurinol ( $5 \mathrm{mg} / \mathrm{kg} /$ day) was used as a positive control. (a) AEPP treatment at $100 \mathrm{mg} / \mathrm{kg}$ and $200 \mathrm{mg} / \mathrm{kg}$ reversed MSU-induced increases in MPO activity in synovial liquid. (b) MEPP treatment at $100 \mathrm{and} 200 \mathrm{mg} / \mathrm{kg}$ reversed MSUinduced increases in MPO activity in synovial liquid. Each bar represents the mean \pm SEM of 6 individual rats. ${ }^{* *} p<0.01,{ }^{* * *} p<0.001$; significant difference compared to the negative control group (MSU + DMSO) using one-way ANOVA with Tukey’s posttest. ${ }^{\mathfrak{E E}} p<0.001$ significant difference compared to the sham group using one-way ANOVA with Tukey's posttest.

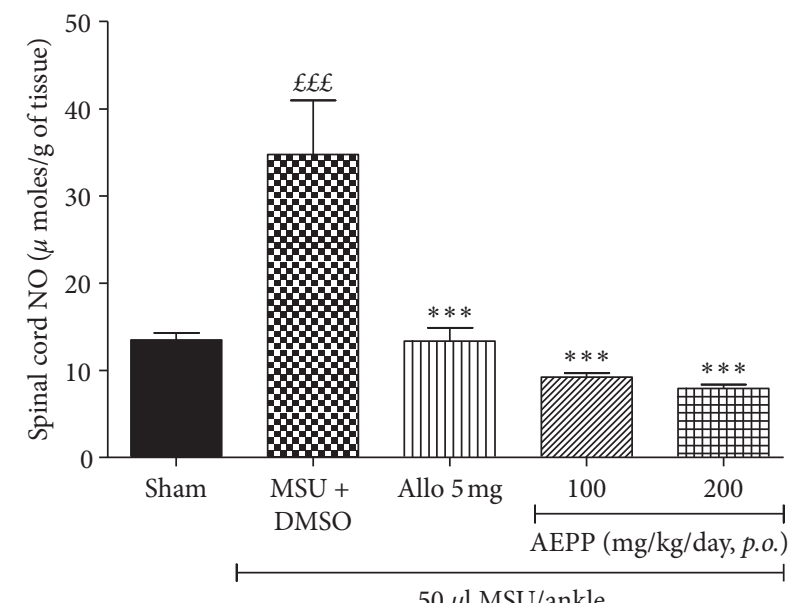

(a)

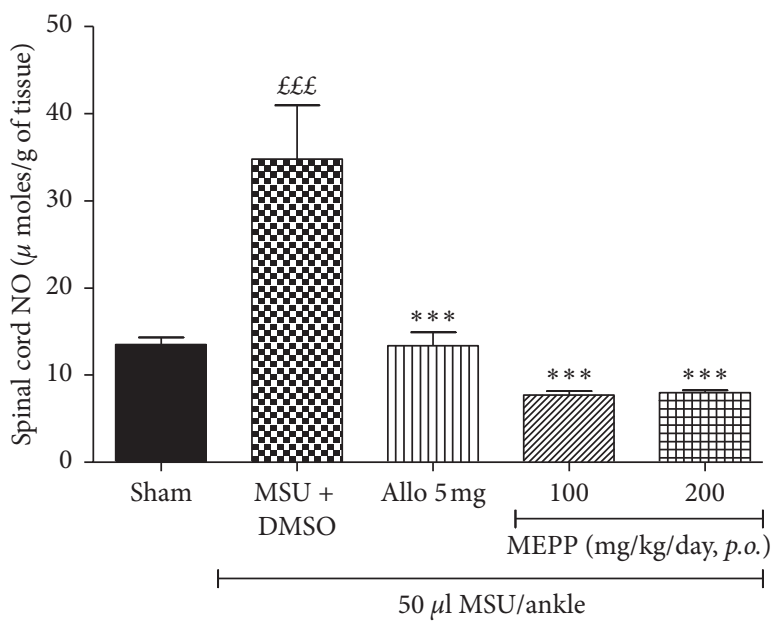

(b)

Figure 4: Continued. 


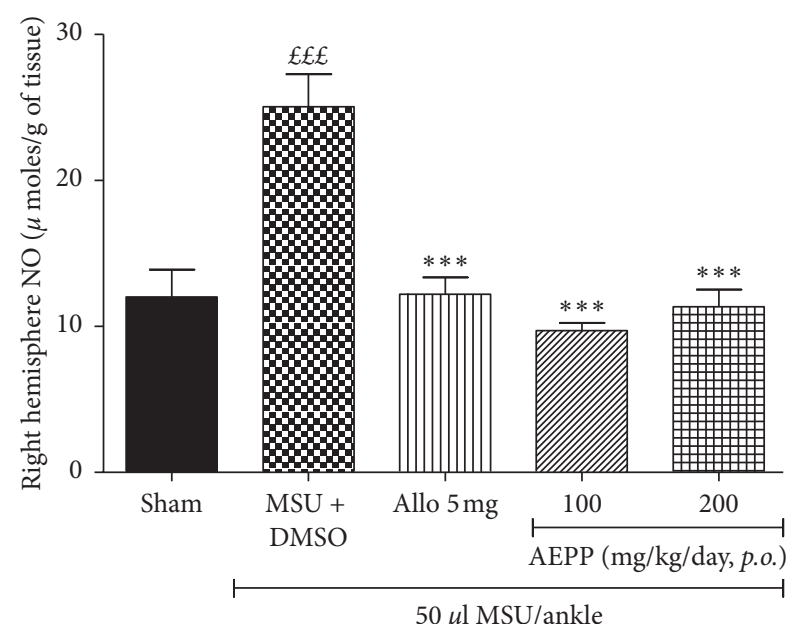

(c)

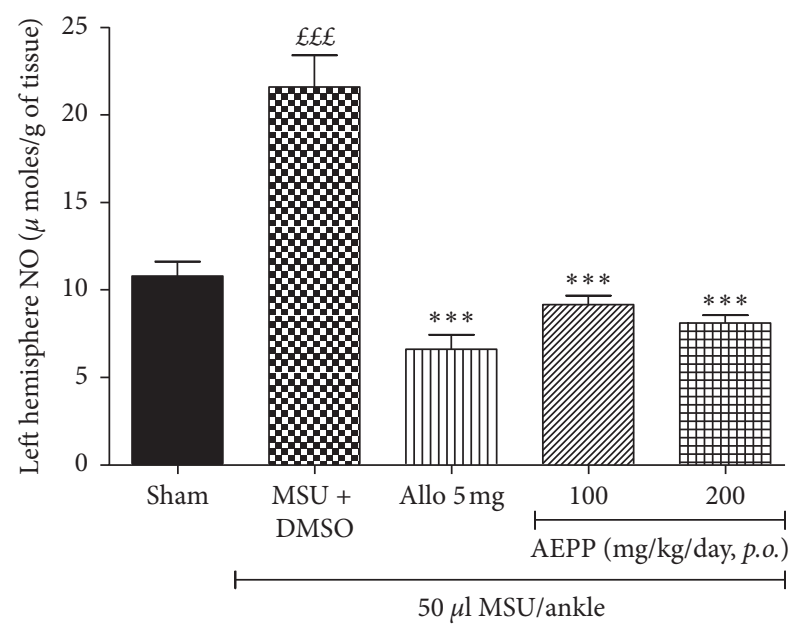

(e)

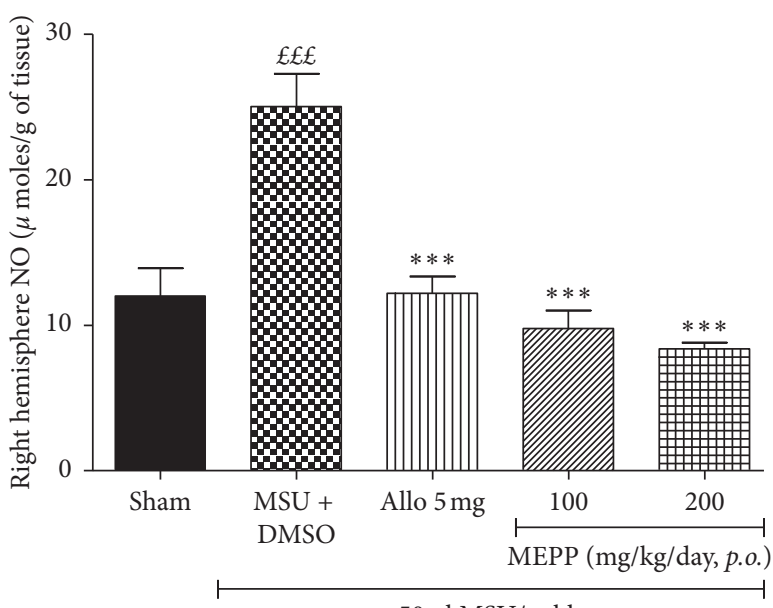

(d)

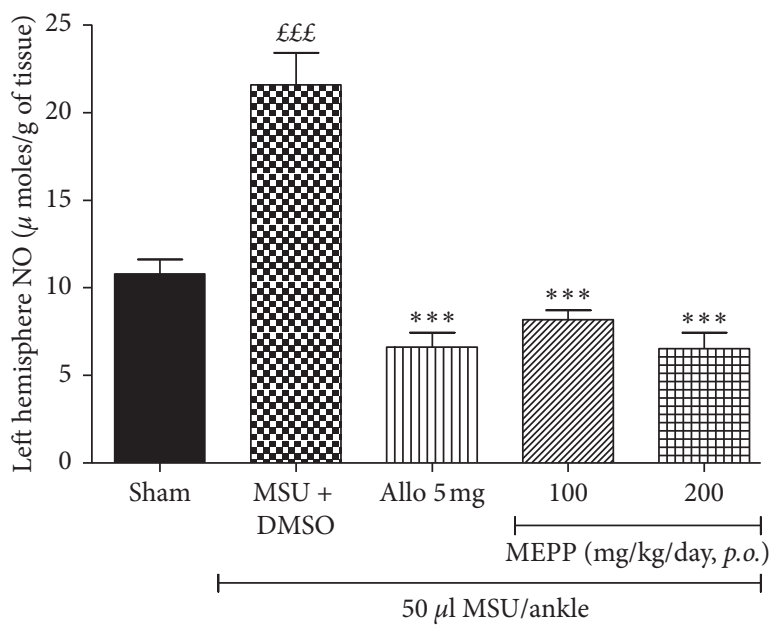

(f)

FIGURE 4: Effects of the aqueous (AEPP) and methanolic (MEPP) extracts of Paullinia pinnata on the nitrite oxide level in the spinal cord (a, b), the right hemisphere $(c, d)$, and the left hemisphere $(e, f)$. Plant extract treatment in all the organs used significantly reversed the overproduction of nitric oxide (NO) induced by the intra-ankle injection of MSU. Each bar represents the mean \pm SEM of five repetitions. ${ }^{* * *} p<0.001$ significant difference compared to the negative control group (MSU + DMSO) using one-way ANOVA with Tukey's posttest. EEE $p<0.001$ significant difference compared to the sham group using one-way ANOVA with Tukey's posttest.

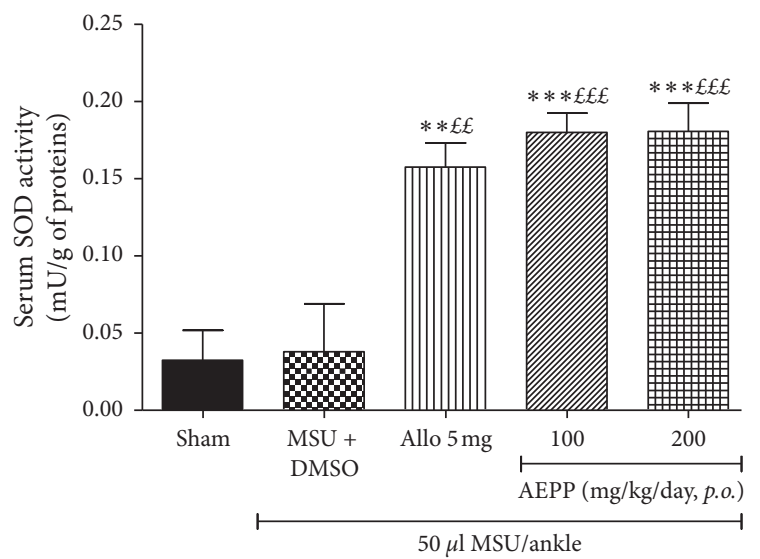

(a)

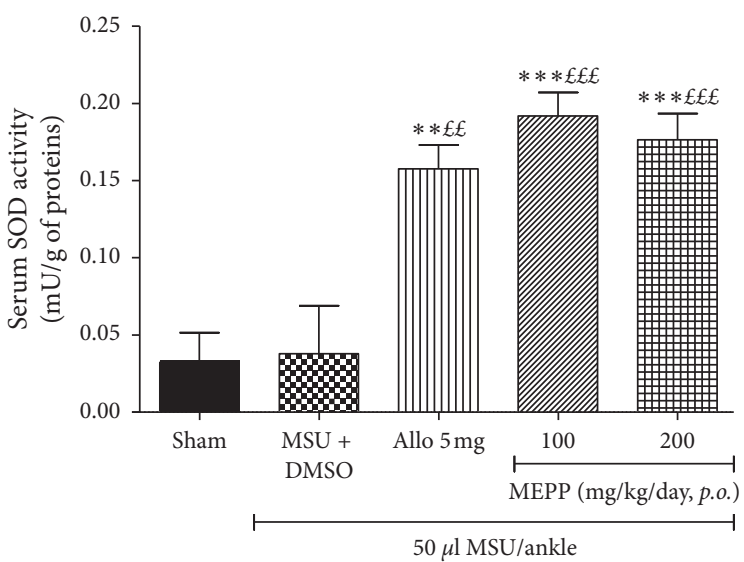

(b)

Figure 5: Continued. 


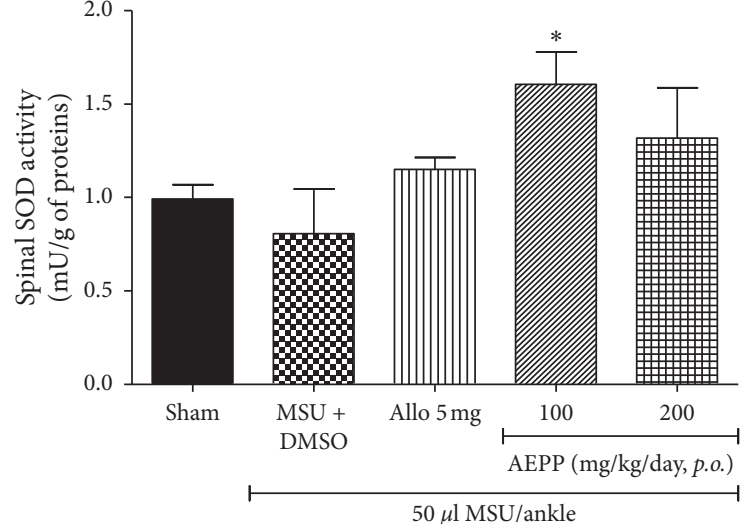

(c)

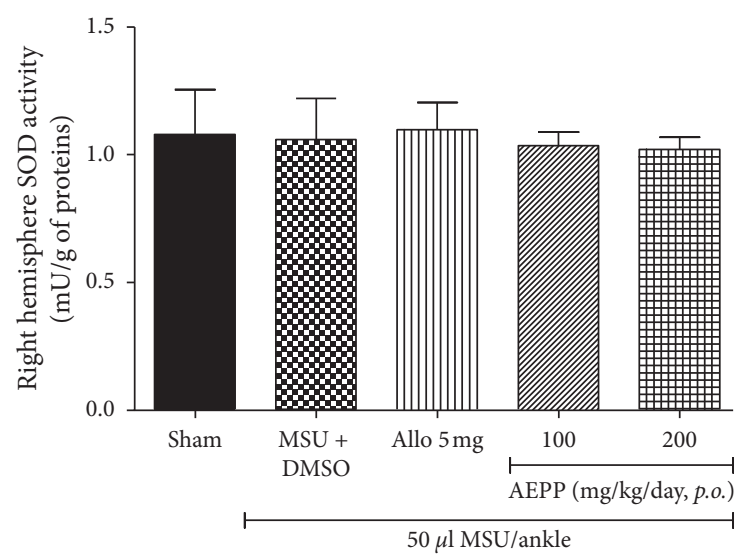

(e)

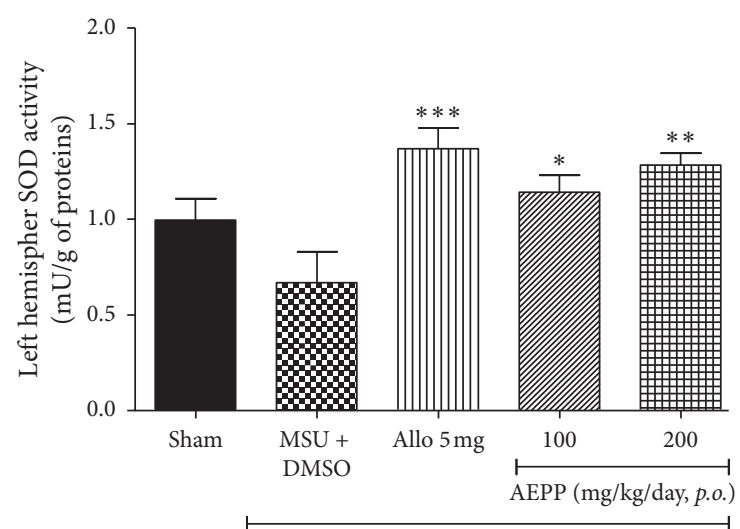

$50 \mu \mathrm{l} \mathrm{MSU} / \mathrm{ankle}$

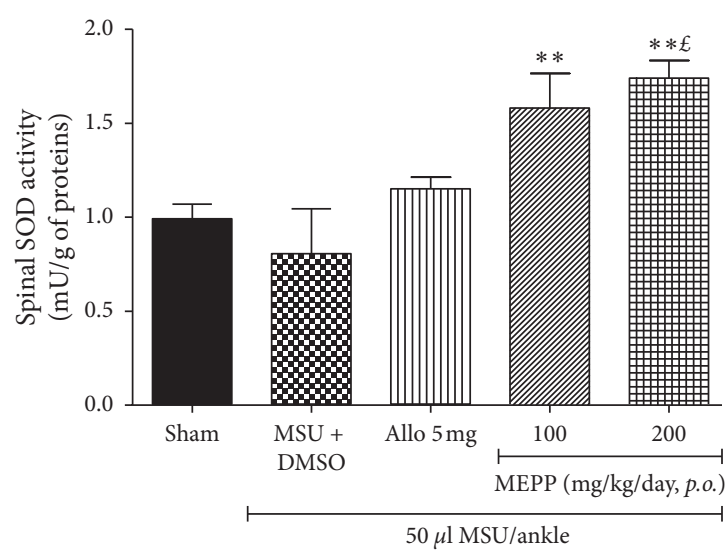

(d)

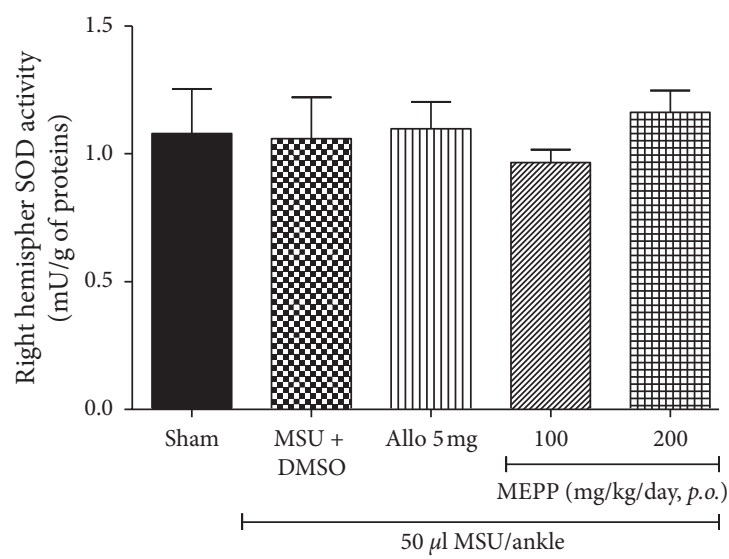

(f)

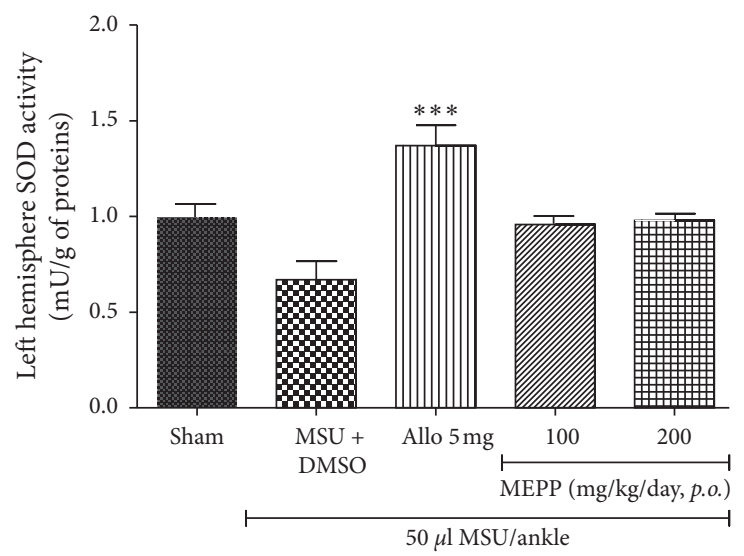

(h)

FIGURE 5: Effects of the aqueous (AEPP) and methanol (MEPP) extracts of Paullinia pinnata on the superoxide dismutase (SOD) activity in the serum (Figures 4(a) and 4(b)), spinal cord (Figures 4(c) and 4(d)), right hemisphere (Figures 5(e and f)), and left hemisphere (Figures 5(g and h)) of MSU-injected rats. Allopurinol $(5 \mathrm{mg} / \mathrm{kg} /$ day) serves as a positive control for antigouty effects. SOD activity significantly increased after treatment with allopurinol, AEPP (100 and 200), and MEPP (100 and 200) in the serum (a and b). Only the AEPP 100 and the MEPP (100 and 200) significantly increase SOD activity in the spinal cord (c and d). Both the AEPP (100 and 200) and the MEPP (100 and 200) fail to increase SOD activity in the right hemisphere (e and f). The AEPP 200 and allopurinol significantly reverse MSU-induced SOD exhaustion in the left hemisphere ( $\mathrm{g}$ and $\mathrm{h}$ ). Each bar represents the mean \pm SEM of 6 individual rats, ${ }^{*} p<0.05,{ }^{* *} p<0.01,{ }^{* * *} p<0.001$; significant difference compared to the negative control group (MSU + DMSO) using one-way ANOVA with Tukey's posttest. ${ }^{\mathfrak{E}} p<0.05$, $\mathfrak{E E}^{\mathfrak{E}} p<0.01$, ${ }^{\mathfrak{E E}} p<0.001$; significant difference compared to the sham group using one-way ANOVA with Tukey's posttest.

among which are myeloperoxidases (MPO). To determine the effects of $P$. pinnata extract on neutrophils infiltration and activation, we evaluated the MPO activity in the synovial fluid. Both AEPP and MEPP potently reduced the MPO activity, confirming that these extracts reduce cell infiltration as previously demonstrated [16]. Also, the possibility that $P$. 


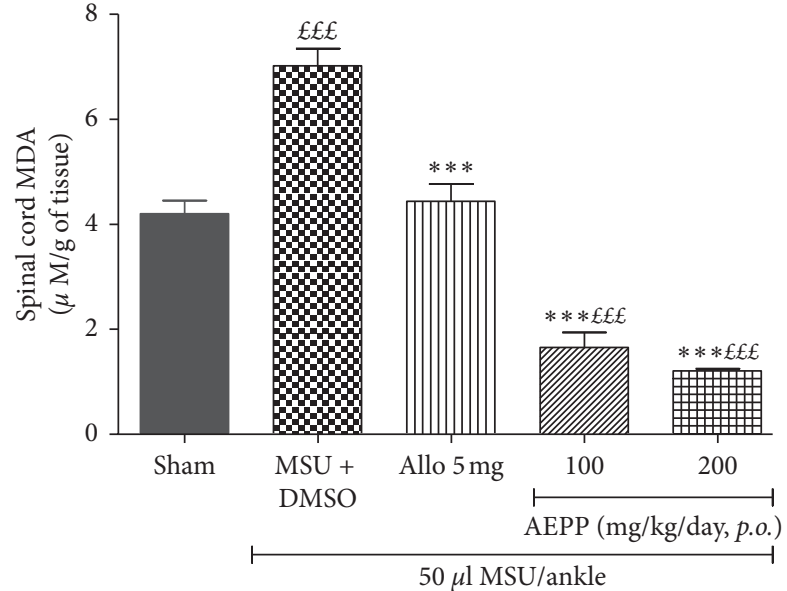

(a)

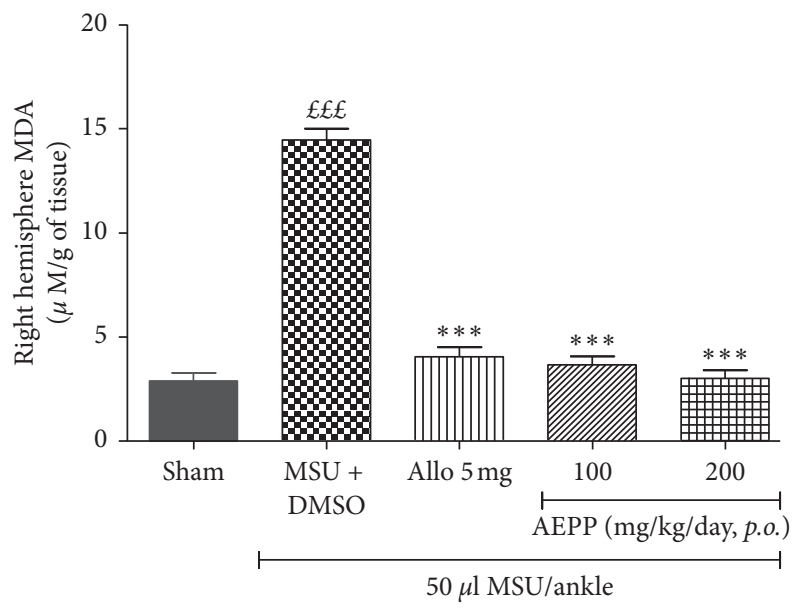

(c)

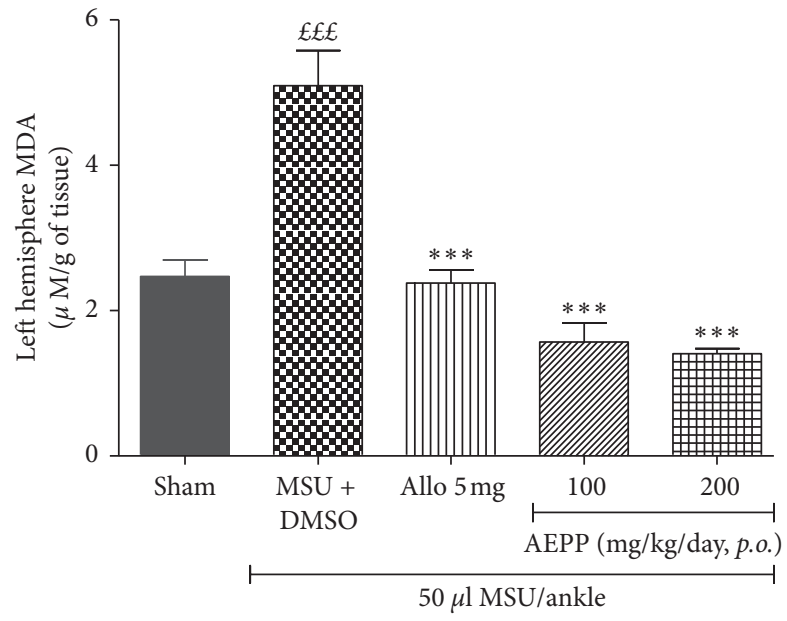

(e)

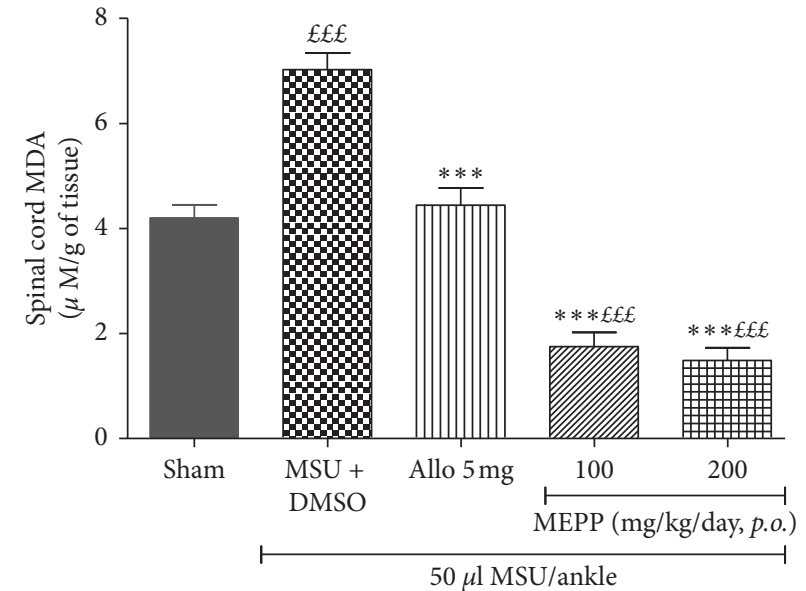

(b)

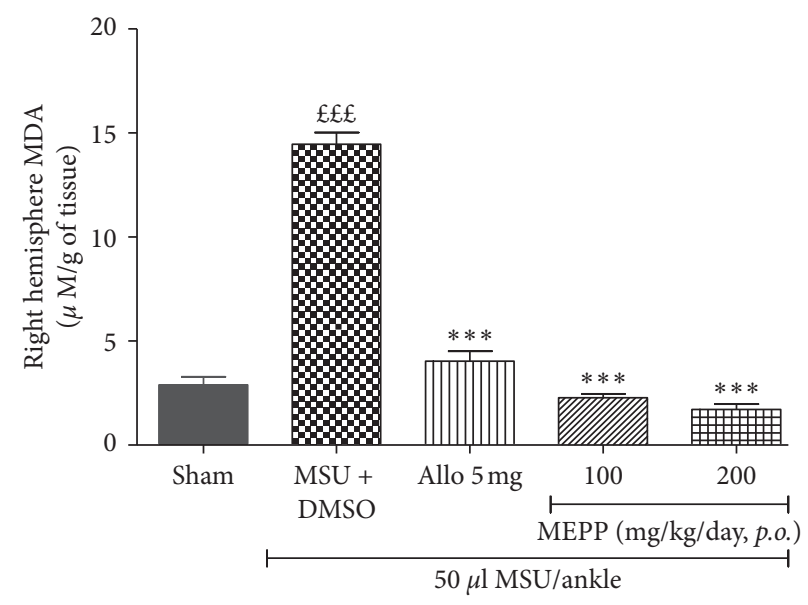

(d)

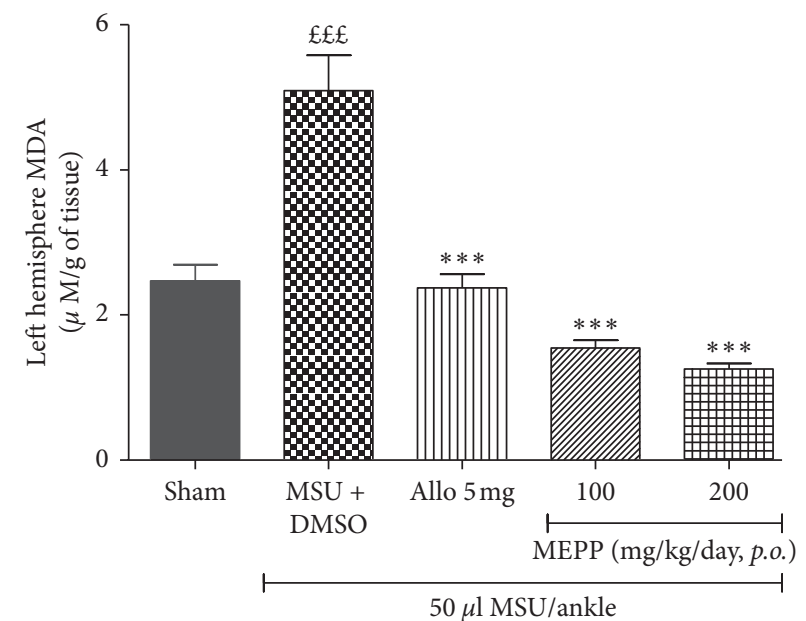

(f)

FIGURE 6: Effects of the aqueous (AEPP) and methanol (MEPP) extracts of Paullinia pinnata on the malondialdehyde (MDA) content in the spinal cord of MSU-injected animals. Allopurinol ( $5 \mathrm{mg} / \mathrm{kg} /$ day) is used as a positive control for antigouty effects. (a) AEPP treatment at $200 \mathrm{mg} / \mathrm{kg}$ and $300 \mathrm{mg} / \mathrm{kg}$ reversed MSU-induced increases in MDA spinal cord content (one-way ANOVA significant main effect $p<0.001$ ). (b) MEPP treatment at 100, 200, and $300 \mathrm{mg} / \mathrm{kg}$ reversed MSU-induced increase in MDA spinal cord content (one-way ANOVA significant main effect $p<0.001$ ). Each bar represents the mean \pm SEM of 6 individual rats. ${ }^{* * *} p<0.001$; significant difference compared to the negative control group (MSU + DMSO) using one-way ANOVA with Tukey’s posttest. ${ }^{£} p<0.05,{ }^{\mathfrak{E}} p<0.01,{ }^{\mathfrak{E} \in} p<0.001 ;$ significant difference compared to sham using one-way ANOVA with Tukey's posttest. 
pinnata extracts could inhibit the release of inflammatory mediators from leucocytes cannot be rolled out, as previously discussed. It has been shown that the methanol extract of the leaves of $P$. pinnata possesses $\beta$-sitosterol and friedelin [14]. The analgesic and the anti-inflammatory properties of these compounds have been demonstrated $[28,29]$. Therefore, it can be assumed that the analgesic and anti-inflammatory effects of the methanol extract of $P$. pinnata is related to the presence of $\beta$-sitosterol and friedelin.

One of the early events associated with gout is the oxidative stress [30] at the level of the affected joint. Besides, the constant transmission of noxious stimulus in the spinal cord and the brain, promotes the release of reactive oxygen species (ROS), leading to oxidative stress in these tissues. It has been demonstrated that ROS mediate the development and maintenance of pain and further boost the peripheral and central sensitization in joint pain [30]. Concordantly, it was observed in the present study that MSU-induced gout was accompanied by substantial production of $\mathrm{NO}$ and malondialdehyde in the spinal cord and the brain while the superoxide dismutase content was not significantly affected. These data confirm the oxidative status in the brain and spinal cord of MSU gouty animals. AEPP and MEPP significantly reduced both NO and MDA contents and increased the SOD activity in the tissues, further confirming the in vivo antioxidant effects of $P$. pinnata extracts as previously suggested [16].

\section{Conclusion}

The present study shows that the aqueous and methanol extracts from the leaves of $P$. pinnata possess antihyperalgesic and anti-inflammatory effects against MSUinduced gouty arthritis. The analgesic effect is at least partially related to its anti-inflammatory activity. The antiinflammatory effect seems to be due to the inhibition of the inflammatory cells infiltration and/or the inactivation of infiltrated cells. The antioxidant effect of these extracts also highly contributes to their antigouty activity. These results support the use of the leaves of $P$. pinnata extracts in Cameroonian folk medicine for the management of gouty arthritis.
Abbreviation
MSU: Monosodium urate
AEPP: Aqueous extract Paullinia pinnata
MEPP: Methanol extract Paullinia pinnata
$\mathrm{NaOH}$ : Sodium hydroxide
$\mathrm{HCl}$ : Hydrogen chloride
DMSO: Dimethyl-sulfoxide
MDA: Malondialdehyde
NO: $\quad$ Nitrite oxide
SOD: Superoxide dismutase
MPO: Myeloperoxidase
ROS: Reactive oxygen species
CFA: Complete Freud's Adjuvant.

\section{Data Availability}

The data used to support the findings of this study are available from the corresponding author upon request.

\section{Ethical Approval}

All procedures used in the present study were approved by the local Ethical Committee (FS, University of Dschang) and conformed to the guidelines for the study of pain in awake animals established by the International Association for the Study of Pain (protocol number PP00032).

\section{Conflicts of Interest}

The authors declare no conflicts of interest.

\section{Authors' Contributions}

TBN planned the work. PPT, BNP, and SMN collected the data. TBN and PPT carried out the statistical analysis. PPT and TBN drafted the manuscript. All the authors corrected and approved the final copy of the manuscript.

\section{Acknowledgments}

The authors gratefully appreciate the support of the Alexander von Humboldt Foundation (Germany) through the equipment subsidy.

\section{References}

[1] V. H. Sato, B. Sungthong, P.-O. Rinthong et al., "Pharmacological effects of Chatuphalatika in hyperuricemia of gout," Pharmaceutical Biology, vol. 56, no. 1, pp. 76-85, 2018.

[2] C. Chen, J.-M. Lü, and J.-M. Lü, "Hyperuricemia-related diseases and xanthine oxidoreductase (XOR) inhibitors: an overview," Medical Science Monitor, vol. 22, pp. 2501-2512, 2016.

[3] G. Mancia, G. Grassi, and C. Borghi, "Hyperuricemia, urate deposition and the association with hypertension," Current Medical Research Opinion, vol. 31, no. 2, pp. 1-19, 2015.

[4] H. L. Pisaniello, S. Lester, D. Gonzalez-Chica et al., "Gout prevalence and predictors of urate-lowering therapy use: results from a population-based study," Arthritis Research \& Therapy, vol. 20, no. 1, p. 143, 2018.

[5] C. A. Scirè, C. Rossi, L. Punzi, A. Genderini, C. Borghi, and W. Grassi, "Change gout: how to deal with this "silentlydeveloping killer" in everyday clinical practice," Current Medical Research and Opinion, vol. 34, no. 8, pp. 1411-1417, 2018.

[6] Y. Zamudio-Cuevas, K. Martínez-Flores, J. Fernández-Torres et al., "Monosodium urate crystals induce oxidative stress in human synoviocytes," Arthritis Research \& Therapy, vol. 18, p. 117, 2016.

[7] F. I. A. Bakar, M. F. A. Bakar, A. Rahmat, N. Abdullah, S. F. Sabran, and S. Endrini, "Anti-gout potential of Malaysian medicinal plants," Frontiers in Pharmacology, vol. 9, p. 261, 2018.

[8] M. Umamaheswari, K. Asokkumar, A. T. Sivashanmugam, A. Remyaraju, V. Subhadradevi, and T. K. Ravi, "In vitro xanthine oxidase inhibitory activity of the fractions of 
erythrina stricta Roxb," Journal of Ethnopharmacology, vol. 124, no. 3, pp. 646-648, 2009.

[9] B. N. Cronstein and R. Terkeltaub, "The inflammatory process of gout and its treatment," Arthritis Research \& Therapy, vol. 8, no. 1, p. S3, 2006.

[10] S. Chabra, R. Makuna, and E. Mshiu, "Plant used in traditional medicine in Eastern Tanzania," Journal of Ethnopharmacology, vol. 33, no. 1-2, pp. 143-157, 1991.

[11] A. Zamble, M. Carpentier, A. Kandoussi et al., "Paullinia pinnata extracts rich in polyphenols promote vascular relaxation via endothelium-dependent mechanisms," Journal of Cardiovascular Pharmacology, vol. 47, no. 4, pp. 599-608, 2006.

[12] E. A. Abourashed, N. J. Toyang, J. Choinski Jr., and J. A. Khan, "Two new flavone glycosides from Paullinia pinnata," Journal of Natural Products, vol. 62, no. 8, pp. 1179-1181, 1999.

[13] E. Dongo, H. Hussain, S. R. Miemanang, D. Tazoo, B. Schulz, and K. Krohn, "Chemical constituents of Klainedoxa gabonenses and Paullinia pinnata," Records of Natural Products, vol. 3, no. 3, pp. 165-169, 2009.

[14] K. P. Lunga, X.-J. Qin, X. W. Yang, J.-R. Kuiate, Z. Z. Du, and D. Gatsing, "Antimicrobial steroidal saponin and oleananetype triterpenoidsaponins from Paullinia pinnata," Biomed Central, Complementary and Alternative Medicine, vol. 14, no. 1, p. 369,2014

[15] M. O. Nafiu, S. S. Olaniyi, M. O. Salawu, A. A.-n. Ajao, and M. A. Akanji, "Studies on ameliorative effects of polyphenolic extract from Paullinia pinnata L. (Sapindaceae) on carbon tetrachloride-induced hepatotoxicity and oxidative stress: an in vivo assessment," Notulae Scientia Biologicae, vol. 10, no. 1, pp. 79-86, 2018.

[16] P. P. Tseuguem, D. A. M. Ngangoum, J. M. Pouadjeu et al., "Aqueous and methanol extracts of Paullinia pinnata L. (Sapindaceae) improve inflammation, pain and histological features in CFA-induced mono-arthritis: evidence from in vivo and in vitro studies," Journal of Ethnopharmacology, vol. 236, pp. 183-195, 2019.

[17] N. S. Tank, K. R. Rathod, B. B. Parekh, K. D. Parikh, and M. J. Joshi, "Synthesis and characterization of monosodium urate (MSU) nano particles," American Institute of Physics: Conference Proceedings, vol. 1728, Article ID 020224, p. 4, 2016.

[18] Z. Zhang, H. Xu1, S. Li, W. Zhang, and S. Shi, "Effects of several natural extracts on experimental gouty arthritis in rats," International Conference on Nutrition and Food Sciences, IPCBEE, vol. 39, pp. 12-16, 2012.

[19] B. A. Wandji, F. D. T. Bomba, P. A. Nkeng-Efouet, B. N. Piegang, A. Kamanyi, and T. B. Nguelefack, "Antihyperalgesic activity of the aqueous and methanol extracts of the leaves of Pittosporum mannii hook on CFA-induced persistent inflammatory pain," Inflammopharmacology, vol. 26, no. 1, pp. 197-205, 2018.

[20] M. F. Garça, M. Aslan, B. Tuna, A. Kozan, and H. Cankaya, "Serum myeloperoxidase activity, total antioxidant capacity and nitric oxide levels in patients with chronic otitis media," The Journal of Membrane Biology, vol. 246, no. 7, pp. 519-524, 2013.

[21] R. Torres, L. Macdonald, S. D. Croll et al., "Hyperalgesia, synovitis and multiple biomarkers of inflammation are suppressed by interleukin 1 inhibition in a novel animal model of gouty arthritis," Annals of the Rheumatic Diseases, vol. 68, no. 10, pp. 1602-1608, 2009.

[22] K. W. Ruiz-Miyazawa, L. Staurengo-Ferrari, S. S. Mizokami et al., "Quercetin inhibits gout arthritis in mice: induction of an opioid-dependent regulation of inflammasome," Inflammopharmacology, vol. 25, no. 5, pp. 555-570, 2017.

[23] A. Marcotti, A. Miralles, E. Dominguez et al., "Joint nociceptor nerve activity and pain in an animal model of acute gout and its modulation by intra-articular hyaluronan," Pain, vol. 159, no. 4, pp. 739-748, 2018.

[24] K. A. Sluka, W. D. Willis, and K. N. Westlund, "The role of dorsal root reflexes in neurogenic inflammation," Pain Forum, vol. 4, no. 3, pp. 141-149, 1995.

[25] F. A. Pinho-Ribeiro, W. A. Verri Jr., and I. M. Chiu, "Nociceptor sensory neuron-immune interactions in pain and inflammation," Trends in Immunology, vol. 38, no. 1, pp. 5-19, 2017.

[26] Y. C. Lee, N. J. Nassikas, and D. J. Clauw, "The role of the central nervous system in the generation and maintenance of chronic pain in rheumatoid arthritis, osteoarthritis and fibromyalgia," Arthritis Research \& Therapy, vol. 13, no. 2, p. 211, 2011.

[27] A. So, "How to regulate neutrophils in gout," Arthritis Research \& Therapy, vol. 15, no. 5, p. 118, 2013.

[28] S. A. Nirmal, S. C. Pal, S. C. Mandal, and A. N. Patil, "Analgesic and anti-inflammatory activity of $\beta$-sitosterol isolated from Nyctanthes arbortristis leaves Nyctanthes arbortristis leaves," Inflammopharmacology, vol. 20, no. 4, pp. 219-224, 2012.

[29] P. Antonisamy, V. Duraipandiyan, and S. Ignacimuthu, "Anti-inflammatory, analgesic and antipyretic effects of friedelin isolated from azima tetracantha lam. in mouse and rat models," Journal of Pharmacy and Pharmacology, vol. 63, no. 8, pp. 1070-1077, 2011.

[30] Y. Zamudio-Cuevas, C. Hernández-Díaz, C. Pineda et al., "Molecular basis of oxidative stress in gouty arthropathy," Clinical Rheumatology, vol. 34, no. 10, pp. 1667-1672, 2015. 


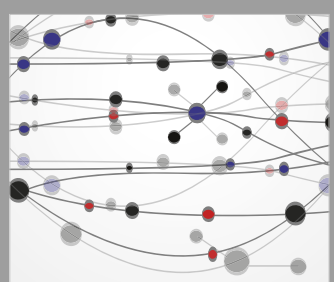

The Scientific World Journal
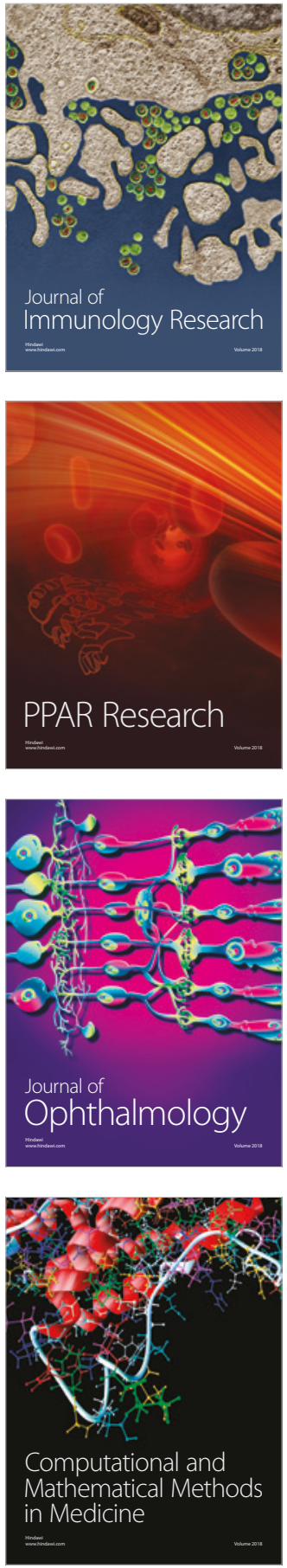

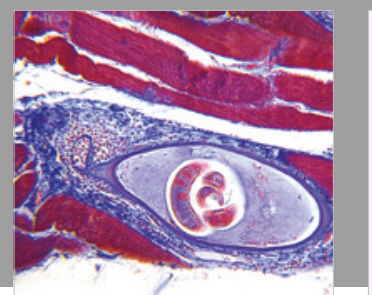

Gastroenterology Research and Practice

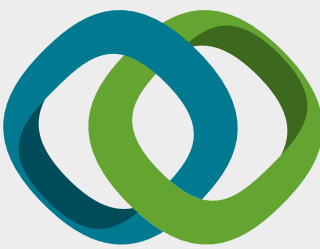

\section{Hindawi}

Submit your manuscripts at

www.hindawi.com
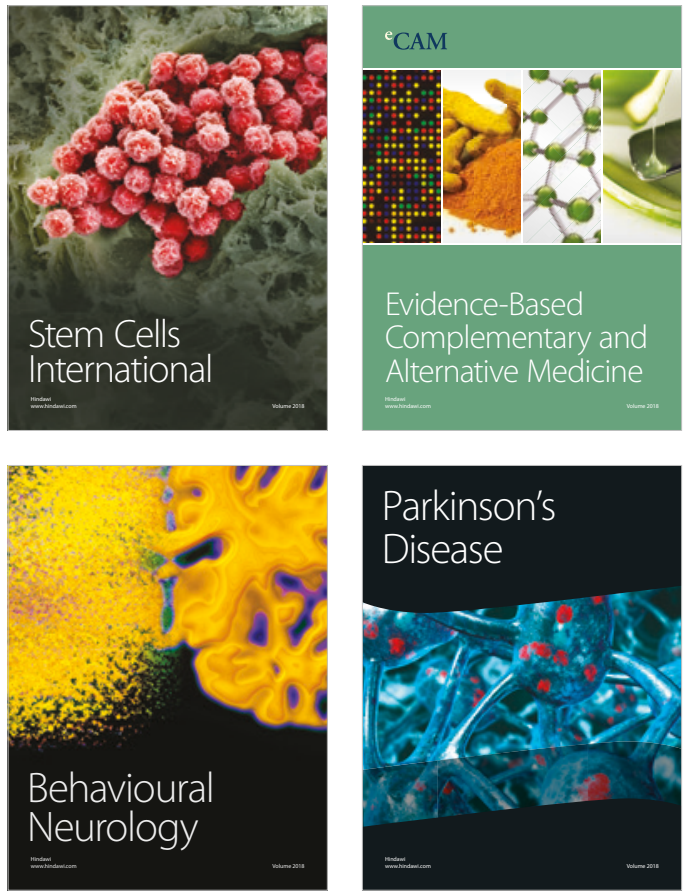

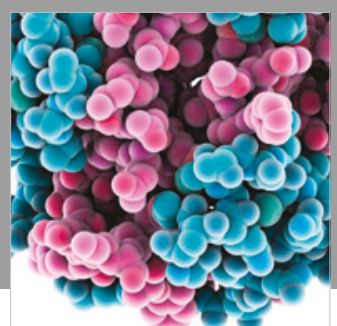

ournal of

Diabetes Research

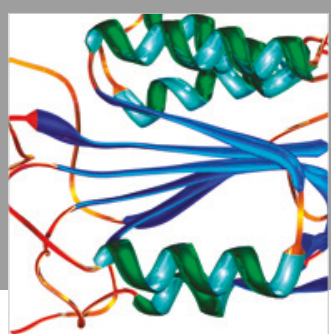

Disease Markers
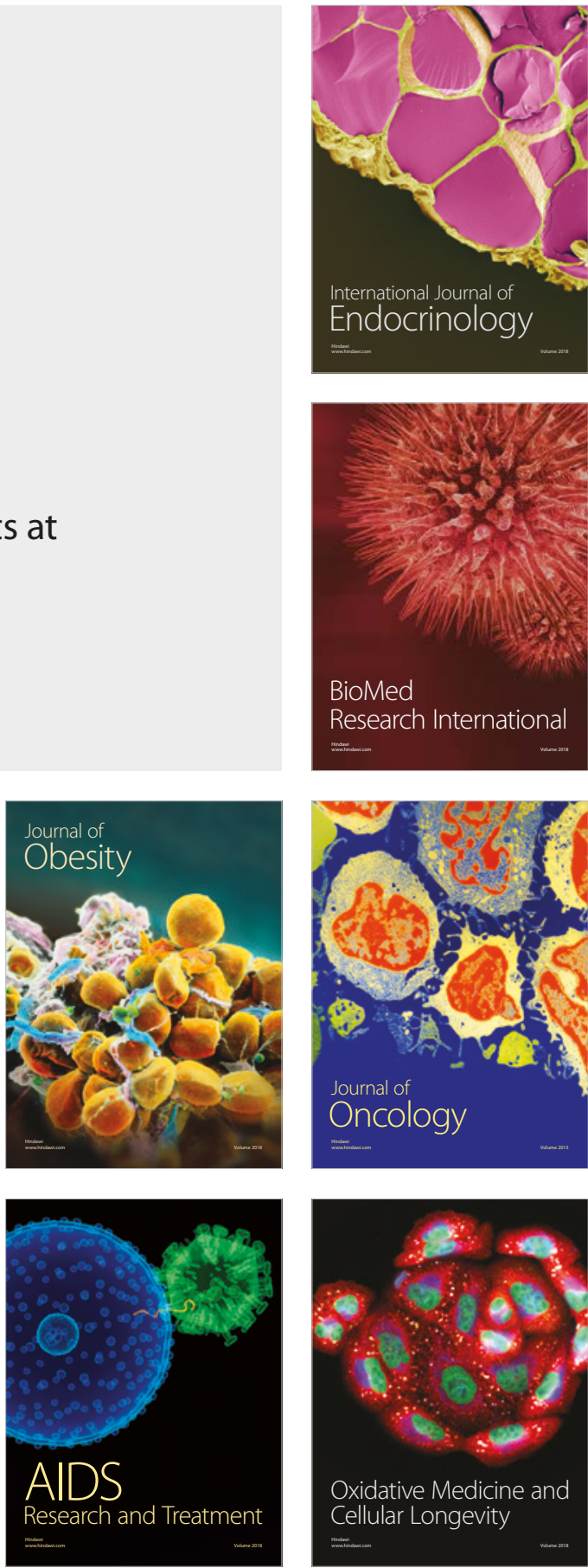